\title{
Association of GUTB and Tubercular inguinal lymphadenopathy - A rare co-occurrence.
}

\author{
${ }^{1}$ Hemant Kamal, ${ }^{2}$ Dr. Kirti Kshetrapal, ${ }^{3}$ Dr. Hans Raj Ranga \\ ${ }^{1}$ Professor, Department of Urology \& reconstructive surgery, PGIMS Rohtak-124001 \\ (Haryana) Mobile- 9215650614 \\ ${ }^{2}$ Prof. Anaesthesia PGIMS Rohtak, \\ ${ }^{3}$ Associate Prof. Surgery PGIMS Rohtak.
}

\begin{abstract}
Here we present a rare combination of GUTB with B/L inguinal lymphadenopathy in a $55 y$ old male patient presented with pain right flank, fever \& significant weight loss for the last 3 months. Per abdomen examination revealed non-tender vague lump in right lumber region about $5 \times 4 \mathrm{~cm}$ dimensions, with $B / L$ inguinal lymphadenopathy, firm, matted. Investigations revealed low haemoglobin count, high leucocytic \& ESR count, urine for AFB was positive and ultrasound revealed small right renal \& psoas abscess, which on subsequent start of ATT, got resolved and patient was symptomatically improved.
\end{abstract}

\section{Introduction}

Genitourinary tuberculosis (GUTB) is the second most common form of extrapulmonary tuberculosis after lymph node involvement [1]. Most studies in peripheral LNTB have described a female preponderance, while pulmonary TB is more common in adult males [2]. In approximately $28 \%$ of patients with GUTB, the involvement is solely genital [3]. However, the combination of GUTB and LNTB is rare condition. Most textbooks mention it only briefly. This report aims to present a case of GUTB with LNTB in a single patient.

\section{Case Report}

55y male with no comorbidities, having pain right flank \& fever X 3months. There was significant weight loss \& loss of apetite present. Abdomen examination revealed an ill defined, vague lump, size $5 \times 4 \mathrm{~cm}$, palpable in right lumbar region. External genitalia revealed no abnormality, but there were bilateral inguinal lymphadenopathy, non-tender, firm \& matting of lymph node was present ( figure 1 ). Digital rectal examination including proctoscopy was normal. On clinical investigations, patient was found to have severe anemia $(\mathrm{Hb}=6.5)$, counts-13000, ESR-120, urine examination \& culture - both normal, urine for AFB - 2 out of 5 samples were positive for mycobacterium, urine PCR- negative, chest radiography revealed no abnormality, FNAC from bilateral inguinal lymph nodes showed some epitheloid cells granuloma, AFB stain negative, suggestive of tubercular lymphadenopathy, sonography abdomen- $1.8 \mathrm{X} 2.2 \mathrm{~cm}$ collection present at upper pole of right kidney \& $4.2 \times 2.9 \mathrm{~cm}$ abscess seen in right psoas. Contrast CT abdomen ( figure 2 ) showed right psoas muscle bulky with collection in it \& in perinephric area. Left kidney was normal. No retroperitoneal lymphadenopathy seen . Patient was started on 4 drug anti-tubercular drugs in the form of Isoniazid, rifampicin, ethambutol \& pyrazinamide, according to weight of the patient. After 1 month of start of ATT , patient improved symptomatically \& he gained weight . Liver function tests were in normal range, urine for AFB all 5 samples were negative. At 2 months of start of ATT, CT scan abdomen with urography done ( figure 3 ), which showed a wedge shaped hypoattenuated area of size 2.4 X $1.7 \mathrm{~cm}$ in lower pole cortex of right kidney with minimal perinephric fluid collection. After 4 months of start of ATT, patient recovered fully with apetite returning to normal, LFT \& sonography were normal, size of inguinal lymph nodes gradually decreasing in size ( figure 4 ). Patient developed gynaecomastia of right breast ( figure 5 ), which subsided subsequently at the time of discontinuation of ATT at the end of 1 year.

\section{Discussion}

GUTB is still a major health problem in many developing countries including India. Urogenital tuberculosis affects all age ranges, with predominance of males between the ages of 30 and 50 years [4]. Our patient was a 55y old male. The combination of GUTB \& LNTB is extremely rare co-incidence . During search for literature, I could not found any article in which both areas are simultaneously involved. Even primary superficial tuberculous lymphademtis of the groin is a rare condition. It has been noted that in $25 \%$ to $30 \%$ of cases, diagnosis of GUTB is established based on histological patterns and/or by detection of Mycobacterium tuberculosis complex by PCR [5]. In 1940 Thompson [6] reported 324 cases of peripheral lymphadenitis due to tuberculosis, of which 12 showed involvement of the inguinal area. In 4 of the 12 cases (33\% ) -a surprisingly high figure-the inguinal involvement appeared alone. Of the remaining eight patients the 
right side was involved in six and the left in four; in two patients the involvement was bilateral. In 1953 Gale [7] reported on 194 cases of superficial lymphadenitis; $17(8 \%)$ of these involved the inguinal area. The involvement of kidneys, prostate \& testis is primary, while rest other areas are involved secondarily by mycobacterium transferring or directly invading to other urogenital organs. From kidney, infection can enroute to para aortic \& paracaval lymph nodes .Rarely, infection from these retroperitoneal nodes spreads in a retrograde manner to pelvic lymph nodes \& from there to inguinal lymph nodes.Also, one can expect involvement of genitor urinary organs \& inguinal lymph nodes as both primary infection or more rarely perispinous/psoas abscess occasionally points to groin of one or both sides [9], as in our index case . In cases of involvement of lymph node, in a study of "Survey Of Tuberculosis In The Province Of Ontario" By Lawee \& Toronto[9] In 1965, Out Of 56 Cases Of Tubercular Lymphadenitis, 52 Were Having cervical lymphadenopathy, 1 mediastinal, 2 axillary \& 1 inguinal. Kidney involvement commense at level of cortex with formation of micro abscesses, which coalease to form large abscess \& ultimately it burst outside into perinephric space \& inside to involve pelvic calyceal system. It is then AFB are detected in urine. Ususlly, renal involvement is unilateral [8] \& generally without evolution to irreversible renal failure. The involvement of the urinary tract can be insidious \& progress slowly to caseation with ureteric strictures. None of us have seen such a unique case of tubercular involvement of genitourinary tract with lymphatic system . So, we thought that reporting such a case is desirable so that we should be able to know how this infection is going to be a systemic illness, if left untreated.

\section{References}

[1]. Kapoor R, Ansari MS, Mandhani A, Gulia A (2008) Clinical presentation and diagnostic approach in cases of genitor urinary tuberculosis. Indian J Urol 24: 401-405.

[2]. Balasubramanian, R., Ramachandran, R.: Management of non-pulmonary forms of tuberculosis: review of TRC studies over two decades. Indian Journal of Pediatrics 2000;67:S34-S40.

[3]. Shah H, Shah K, Dixit R, Shah KV (2004) Isolated Tuberculous epididymo-orchitis. Indian J Tubercl 51: 159-162

[4]. Figueiredo AA, Lucon AM, Gomes CM, Srougi M (2008) Urogenital Tuberculosis: Patient classification in seven different groups according to clinical and radiological presentation. International Braz J Urol 34: 422-432.

[5]. Cek M, Lenk S, Naber KG, Bishop MC, Bjerklund Johansen TE, Botto H Grabe M, Lobel B, Redorta JP, Tenke P; Members of the Urinary Tract Infection (UTI) Working Group of the European Association of Urology (EAU) Guidelines Office (2005) EAU Guidelines for the Management of Genitourinary Tuberculosis. European Urology 48: 353-362.

[6]. THOMPSON, B. C.: Tubercie 21' 217, 260, 1940.

[7]. GALE, G. L.: Cana4. Med. A88. J., 69: 303. 1953.

[8]. Brenner BM. Urinary tract obstructive disease. In : The kidney. Saunders, Philadelphia : 1991; 1768-1808

[9]. D. LAWEE, M.D., Toronto Primary Tuberculous Inguinal Lymphadenitis : Canad. Med. Ass. J.Jan. 4, 1969, vol. 100

Figure 1

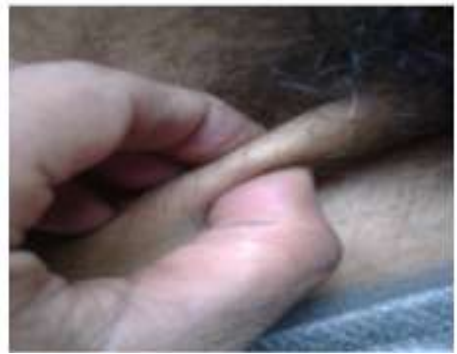

GUTB Figures

Rt. Ing. LAP, matted

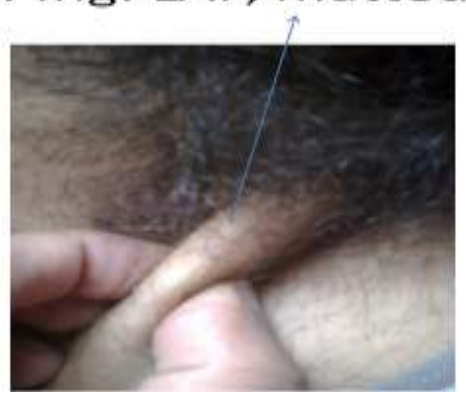

\section{Figure 2}
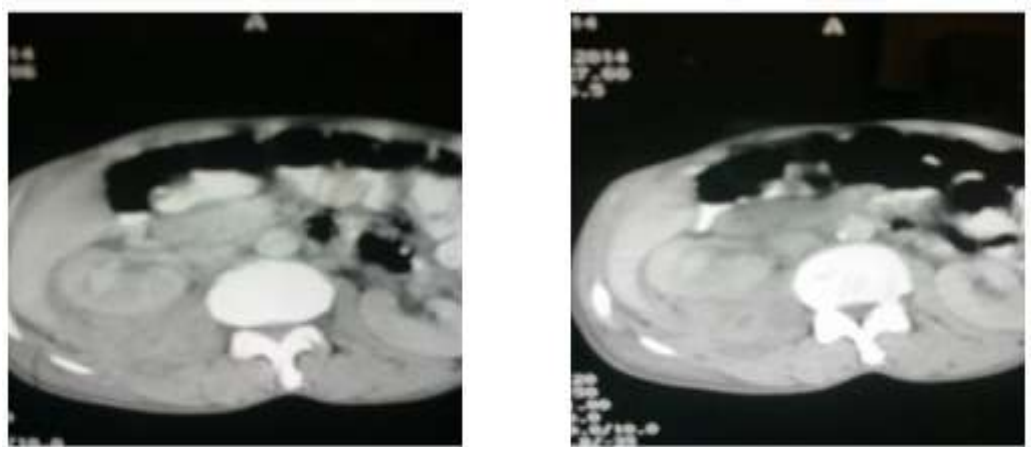


\section{Figure 3}

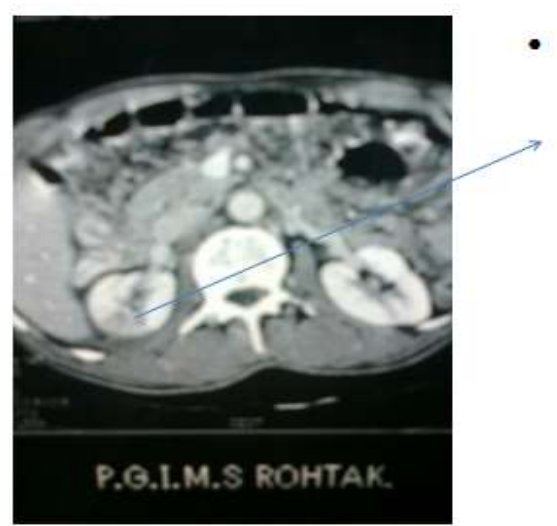

- Hypodense lesion lower pole cortex.

Figure 4

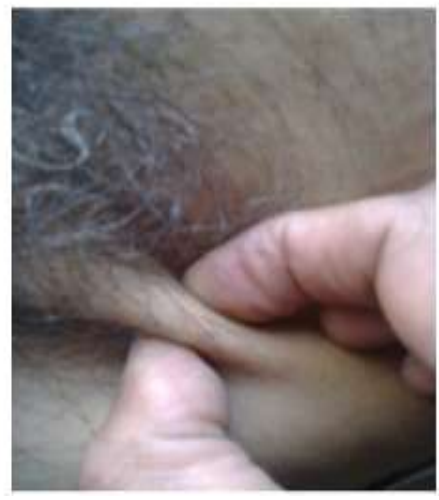

Figure 5

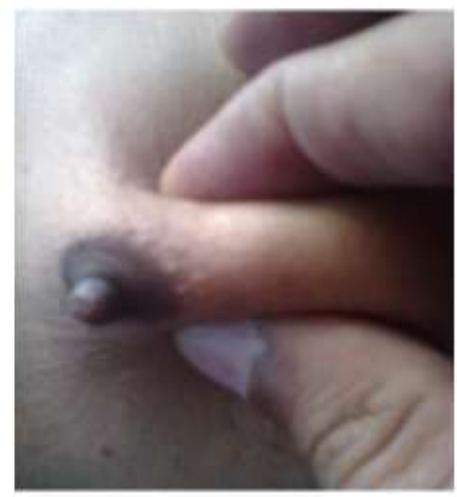

Left ing. LAP after 4M ATT

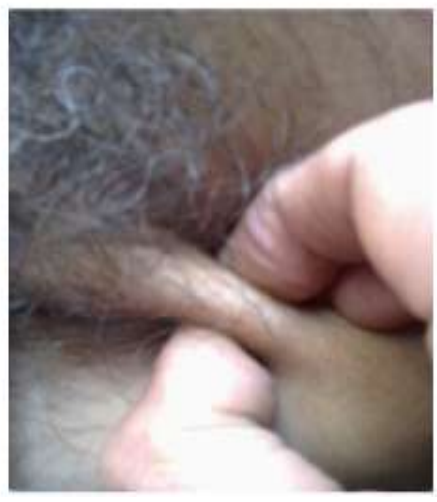

After $5 \mathrm{M}$ of ATT

- Rt. Gynaecomastia, possiblydue to $\mathrm{INH}$. 